\title{
MATHEMATICAL SIMULATION OF DEFORMATION FRICTION FORCE DURING FOOD MATERIAL CUTTING
}

\author{
Oleg Vjatcheslavovich Ageev ${ }^{1}$, Marek Jakubowski², Liviu Giurgiulescu ${ }^{3}$, \\ ${ }^{1}$ Kaliningrad State Technical University, Department of Food and Refrigeration Machines \\ ${ }^{2}$ Koszalin University of Technology, Department of Food Processes and Facilities \\ ${ }^{3}$ North Universitary Center of Baia Mare, Technical University of Cluj Napoca, Chemistry-Biology \\ Department \\ $\otimes_{\text {oleg.ageev@klgtu.ru }}$ \\ https://doi.org/10.34302/crpjfst/2020.12.4.1

\begin{tabular}{ll}
\hline Article history: & ABSTRACT \\
Received: & The process of friction when cutting food materials has been \\
28 April 2020 & investigated theoretically. The muscle tissue of the raw material has \\
Accepted: & been described by the Maxwell-Thomson rheological model. When \\
25 September 2020 & choosing an analytical description of a regular microrelief of food \\
processing equipment knives, taking into account technological & formative factors, a physical-technological theory of surface \\
Keywords: & roughness has been used. A mathematical description of the profile \\
Food cutting; & of the knife rough surface in the form of a dimensionless periodic \\
Friction force; & one-parameter function has been obtained. By solving the \\
Knife edge; & differential equation of viscoelastic material state in a \\
Rheology; & dimensionless form, the law of distribution of dimensionless normal \\
Viscoelasticity & contact pressures over the microprotrusions of the edge rough \\
& surface has been obtained. An expression for the dimensionless \\
& deformation friction force has been obtained. It is established that \\
at speeds tending to zero or infinity, this force tends to zero. The & magnitude of the force increases monotonically with the increase in \\
the measure of the material elasticity and the increase in the & dimensionless length of the knife edge. The dimensionless width of \\
the contact area of microprotrusion is monotonously depends on the & measure of material elasticity and non-monotonously depends on \\
the dimensionless sliding speed with a pronounced minimum.
\end{tabular}

\section{Introduction}

Ensuring resource saving when cutting food provides a thorough analysis of the resistance forces. Reducing the strength of harmful resistance involves minimizing its important component - the deformation force of friction.

The friction force of the food material and the knife surface is a consequence of the edges roughness, which is determined by the parameters of the technological processing of the working body (Grigoriev, 2016). The advanced technology for the production of knives for food processing equipment includes the following main types of operations: machining (finishing turning) and grinding processing, as a result of which the surface profile includes random and systematic components.

Mathematical modeling and experimental study of the friction process of food materials on a rough surface is a current scientific direction in engineering. Spagnoli et al. (2019) have investigated the effect of friction and the blade immersion depth on the resistance force when cutting viscoelastic materials. Also Spagnoli et 
al. (2018) have analyzed the laws of the process of destruction of a viscoelastic material with friction, have analyzed the effect of sharpness of the cutting unit on the distribution of stresses in the material.

Xiao-Ping Zhou et al. (2019) have performed an experimental theoretical analysis of the mechanical behavior of highly elastic media in the process of deformation and fracture. Schuldt et al. (2018) have examined the laws of friction in the food materials processing in a wide range of speeds: from $0.001 \mathrm{~m} / \mathrm{s}$ to 10 $\mathrm{m} / \mathrm{s}$.

Belaasilia et al. $(2017 ; 2018)$ have described numerical modeling of contact phenomena at the separation surface of elastic and rigid bodies. Wang et al. (2016) and Jadav et al. (2018) have investigated the laws of the friction process of various materials when changing the slip mode. Deibel et al. (2014) have analyzed the patterns of cutting multilayer media and have shown the effect of friction forces on the destruction of the material. Kieserling et al. (2018), Winkeljann et al. (2018) have developed modern methods and installations for measuring the tribological characteristics of food products.

However, despite the value of the known works, there is currently no analytical description of the friction forces acting on the working body when cutting food materials. At the same time, to optimize the geometry of the knife according to the criterion of minimum cutting resistance, mathematical modeling of the forces acting on its edges, taking into account the surface roughness, is required.

\section{Materials and methods}

Ageev et al. (2018) have substantiated the choice of rheological models of tissue of viscoelastic food products of animal origin. Differential equations of models with their solutions for three different loading conditions have been considered. It has been established that the fish muscle tissue, until destruction, exhibits a limited flow under load, relaxes under constant load to an equilibrium state, and is fully restored when fully unloaded. It is shown that the results of the experimental testing of the material at low and medium voltages approximately correspond to the three-element Maxwell - Thomson rheological model. (standard viscoelastic body).

\subsection{Mathematical simulation}

When choosing an analytical description of a regular microrelief of the working parts of food processing equipment taking into account technological formative factors, the physicaltechnological theory of surface irregularities has been used (Grigoriev, 2016). The effect of the formative factors in finishing turning is periodic or practically periodic in nature, due to the tool feed, the workpiece turn, the abrasive tool selfsharpening and other conditions. If we assume that the random component is small, then the basis of the microrelief of the knife edge surface is approximately described by a trigonometric polynomial of the form:

$$
f(x)=\frac{a_{0}}{2}+\sum_{n=1}^{p}\left\{a_{n} \cdot \cos \left(\frac{2 \pi \cdot n \cdot x}{S_{0}}+\bar{\psi}_{n}\right)\right\},
$$

where $S_{0}$ - the step of the profile (step of the first harmonic); $p$ - the order of the polynomial (number of harmonics); $a_{n}, \bar{\psi}_{n}-$ the Fourier coefficient and phase angle of the $n$-ith component of the profile; $a_{0} / 2$ - the zero term of the expansion for the profile curve (coordinate of the center line of the profile in such a coordinate system in which the ordinate is parallel to the center line of the profile).

Denote that $a_{0}=A ; a_{1}=0.5 \cdot A$.

A model of a periodic surface profile containing one harmonic is expressed from (1) in the following form:

$$
f(x)=A \cdot \cos ^{2}\left(\frac{\pi \cdot x}{S_{0}}+\bar{\psi}\right) .
$$

Differential equation of the material state:

$$
\frac{d p}{d x} u+p \frac{E_{0}+E_{1}}{\eta}=\frac{d f}{d x} u E_{0}+f \frac{E_{0} \cdot E_{1}}{\eta} \text {, (3) }
$$

where $p-$ contact pressure; $E_{0}$ instantaneous modulus of material elasticity (Young's modulus); $E_{1}$ - delayed modulus of 
material elasticity; $\eta-$ coefficient of dynamic viscosity of material; $u$ - knife speed.

The solution of the differential equation (3) with the initial condition $p(0)=0$ is:

$$
\begin{array}{r}
p(x)=\frac{A \cdot \xi \cdot k}{A_{0} \cdot\left(4 \pi^{2}+k^{2} \cdot S_{0}^{2}\right)} \cdot\left\{-\left(\vartheta+0.5 \cdot k \cdot S_{0}^{2}\right) \cdot \cos \left(\frac{2 \pi \cdot x}{S_{0}}\right)-\right. \\
\left.-\pi \cdot e_{01} \cdot S_{0} \cdot \sin \left(\frac{2 \pi \cdot x}{S_{0}}\right)+0.5 \cdot k \cdot S_{0}^{2}+\frac{2 \pi^{2}}{k}+\left[\vartheta-\frac{2 \pi^{2}}{k}\right] \cdot \exp (k \cdot x)\right\},
\end{array}
$$

where $k=-\frac{E_{0}+E_{1}}{\eta \cdot u} ; \xi=\frac{E_{0} \cdot E_{1}}{E_{0}+E_{1}} ; \vartheta=\frac{2 \cdot \pi^{2} \cdot E_{0}}{\xi \cdot k}=\frac{2 \cdot \pi^{2}}{k} \cdot\left(e_{01}+1\right) ; e_{01}=\frac{E_{0}}{E_{1}}=\left(\frac{E_{0}}{\xi}-1\right)$.

$$
\begin{aligned}
& F_{2}=l_{k} \cdot \int_{0}^{x_{B}}\left(\frac{d f}{d x} \cdot p(x)\right) d x=l_{k} \cdot \int_{0}^{x_{B}}\left\{\frac{\pi \cdot A}{S_{0}} \cdot \sin \left(\frac{2 \pi \cdot x}{S_{0}}\right) \cdot p(x)\right\} d x= \\
&=\left.\frac{\pi \cdot A^{2} \cdot \xi \cdot k \cdot l_{k}}{A_{0} \cdot S_{0} \cdot\left(4 \pi^{2}+k^{2} \cdot S_{0}^{2}\right.}\right) \cdot \int_{0}^{x_{B}}\left\{\operatorname { s i n } ( \frac { 2 \pi \cdot x } { S _ { 0 } } ) \cdot \left[-\left(\vartheta+0.5 \cdot k \cdot S_{0}^{2}\right) \cdot \cos \left(\frac{2 \pi \cdot x}{S_{0}}\right)-\right.\right. \\
&\left.\left.-\pi \cdot e_{01} \cdot S_{0} \cdot \sin \left(\frac{2 \pi \cdot x}{S_{0}}\right)+0.5 \cdot k \cdot S_{0}^{2}+\frac{2 \pi^{2}}{k}+\left(\vartheta-\frac{2 \pi^{2}}{k}\right) \cdot \exp (k \cdot x)\right]\right\} d x .
\end{aligned}
$$

Lets use the Newton-Leibniz formula and obtain from (5) an expression for the deformation friction force:

$$
\begin{gathered}
F_{2}=\frac{\pi \cdot A^{2} \cdot \xi \cdot k \cdot l_{k}}{A_{0}} \cdot J_{1} \cdot\left\{\frac{1}{8 \pi} \cdot\left[I_{1} \cdot \cos \left(\frac{4 \pi \cdot x_{B}}{S_{0}}\right)+\pi \cdot e_{01} \cdot S_{0} \cdot \sin \left(\frac{4 \pi \cdot x_{B}}{S_{0}}\right)\right]+\right. \\
+J_{1} \cdot k \cdot S_{0} \cdot\left(\vartheta-\frac{2 \pi^{2}}{k}\right) \cdot \sin \left(\frac{2 \pi \cdot x_{B}}{S_{0}}\right) \cdot \exp \left(k \cdot x_{B}\right)- \\
-\frac{J_{1}}{2 \pi} \cdot \cos \left(\frac{2 \pi \cdot x_{B}}{S_{0}}\right) \cdot\left[\left(0.5 \cdot k \cdot S_{0}^{2}+\frac{2 \pi^{2}}{k}\right) \cdot \frac{1}{J_{1}}+4 \pi^{2} \cdot\left(\vartheta-\frac{2 \pi^{2}}{k}\right) \cdot \exp \left(k \cdot x_{B}\right)\right]+ \\
\left.+\frac{J_{1}}{2 \pi} \cdot\left[\left(0.5 \cdot k \cdot S_{0}^{2}+\frac{2 \pi^{2}}{k}\right) \cdot \frac{1}{J_{1}}+4 \pi^{2} \cdot\left(\vartheta-\frac{2 \pi^{2}}{k}\right)\right]-0.5 \cdot \pi \cdot e_{01} \cdot x_{B}-\frac{I_{1}}{8 \pi}\right\},
\end{gathered}
$$

where $J_{1}=\frac{1}{\left(4 \pi^{2}+k^{2} \cdot S_{0}^{2}\right)} ; I_{1}=\vartheta+0,5 \cdot k \cdot S_{0}^{2}$. 
Lets introduce the dimensionless velocity $\bar{u}$ and dimensionless coordinate $\bar{x}$ :

$$
u=\bar{u} \cdot \frac{S_{0} \cdot\left(E_{0}+E_{1}\right)}{\eta} ; \quad k=-\frac{1}{\bar{u} \cdot S_{0}} ; \quad J_{1}=\frac{1}{4 \pi^{2}+k^{2} \cdot S_{0}^{2}}=\frac{\bar{u}^{2}}{4 \pi^{2} \cdot \bar{u}^{2}+1}
$$

$I_{1}=-S_{0} \cdot\left(\frac{0.5}{\bar{u}}+2 \pi^{2} \cdot \bar{u} \cdot\left(e_{01}+1\right)\right) ; \frac{x_{B}}{S_{0}}=\bar{x}_{B} ; k \cdot x=-\frac{\bar{x}}{\bar{u}}$. Taking into account $F_{0}=\left(A^{2} \cdot \xi \cdot l_{k}\right) / A_{0} ;$

$F_{2}=\bar{F}_{2} \cdot F_{0}$ we obtain the expression for

the dimensionless deformation force of friction:

$$
\begin{gathered}
\bar{F}_{2}=\frac{J_{1}}{\bar{u}} \cdot\left\{-0.125 \cdot\left[\bar{I}_{1} \cdot \cos \left(4 \pi \cdot \bar{x}_{B}\right)+\pi \cdot e_{01} \cdot \sin \left(4 \pi \cdot \bar{x}_{B}\right)\right]-\right. \\
-2 \pi^{3} \cdot J_{1} \cdot e_{01} \cdot \sin \left(2 \pi \cdot \bar{x}_{B}\right) \cdot \exp \left(-\bar{x}_{B} / \bar{u}\right)- \\
-0.5 \cdot \cos \left(2 \pi \cdot \bar{x}_{B}\right) \cdot\left[\frac{0.5}{\bar{u}}+2 \pi^{2} \cdot \bar{u}+8 \pi^{4} \cdot J_{1} \cdot \bar{u} \cdot e_{01} \cdot \exp \left(-\bar{x}_{B} / \bar{u}\right)\right]+ \\
\left.+0.5 \cdot\left[\frac{0.5}{\bar{u}}+2 \pi^{2} \cdot \bar{u}+8 \pi^{4} \cdot J_{1} \cdot \bar{u} \cdot e_{01}\right]+0.5 \cdot \pi^{2} \cdot e_{01} \cdot \bar{x}_{B}+0.125 \cdot \bar{I}_{1}\right\} .
\end{gathered}
$$

\section{Results and discussions}

Fig. 1 shows the dependence of the dimensionless deformation friction force along the entire knife edge on the dimensionless sliding speed by various dimensionless length of the edge and measure of the material elasticity. Fig. 2 shows the dependence of the dimensionless deformation friction force along the entire knife edge on the dimensionless length of the edge by different sliding speed and measure of elasticity. The contour graph fig. 3 shows the dependence of the dimensionless deformation friction force on the dimensionless length of the edge and the dimensionless sliding speed, the contour plot fig. 4 - from the dimensionless speed and measure of the material elasticity.

Calculations of the dimensionless deformation friction force on the fig. 1-4 are drawn in accordance with the dimensionless length of the edge $\bar{x}_{B}$. Analysis of expressions (6), (7) shows that the dimensional deformation friction force depends on the height of the microprotrusions, the height of the deformable surface layer of the material, the length of the knife edge, the rheological properties of muscle tissue, the speed of sliding and the edge length of the knife.

Fig. 1 demonstrates that the dimensionless deformation friction force is a non-monotonic function of the dimensionless sliding speed. At slip speeds tending to zero or infinity, this force tends to zero. Zero force corresponds to the solution of the problem for indentation of microprotrusions into elastic material, which is characterized by a long modulus of elasticity $\xi$ . The magnitude of the friction force increases significantly with increasing material elasticity.

Fig. 2 shows that the deformation force of friction increases significantly with an increase in the dimensionless length of the knife edge. As fig. 3 illustrates the dependence of the indicated friction force on the length of the edge is monotonous, as well as the dependence on the measure of the material elasticity according to fig. 4.

With the values of the measure of elasticity 5 , dimensionless edge length $10 ; 20 ; 30 ; 50$, values of the maximum deformation force of friction are $19.958 ; 39.585 ; 59.220 ; 98.489$, 
respectively. At the values of dimensionless face length 20; elasticity measures $2 ; 5 ; 8 ; 12$, values of the maximum deformation force of friction

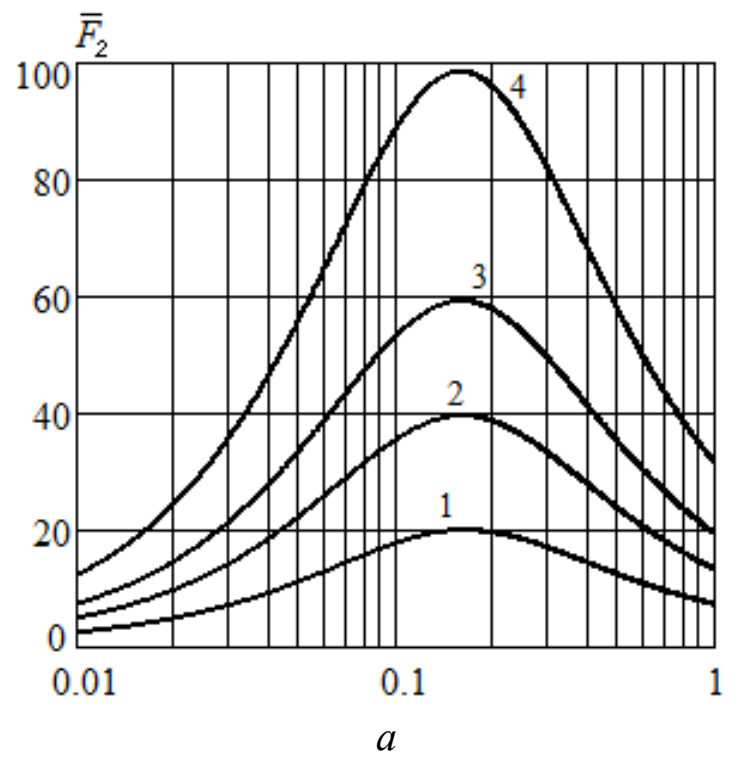

are $15.834 ; 39.585 ; \quad 63.336 ; 95.004$, respectively.

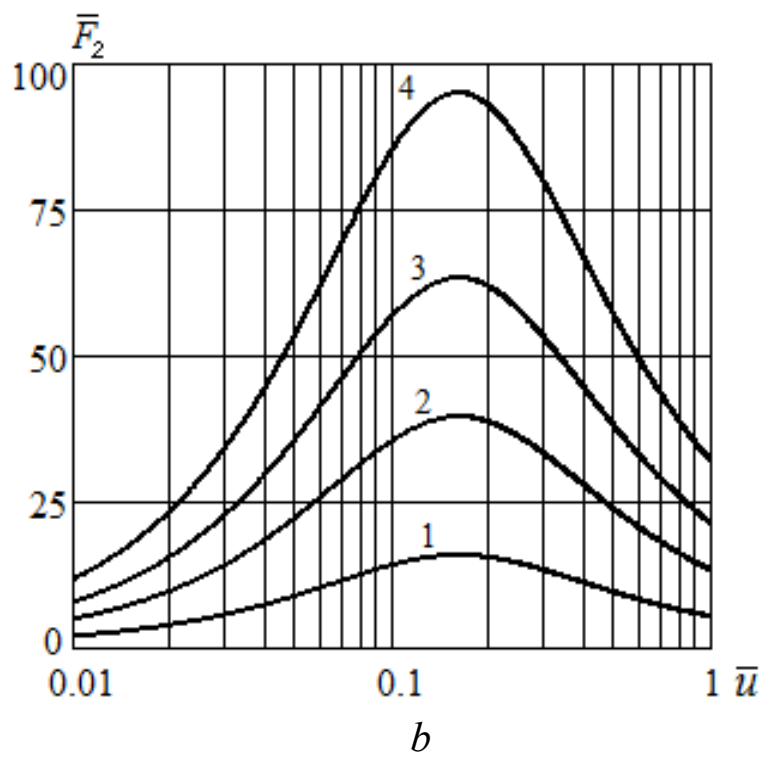

Figure 1. The dependence of the dimensionless friction force on the dimensionless sliding speed (scale $\bar{u}$ - logarithmic): $a$-at various values of the dimensionless length of the edge $\left(e_{01}=5\right)$ :

$$
1-\bar{x}_{B}=10 ; 2-\bar{x}_{B}=20 ; 3-\bar{x}_{B}=30 ; 4-\bar{x}_{B}=50 \text {; }
$$

$b-$ at different values of the measure of material elasticity $\left(\bar{x}_{B}=20\right)$ :

$$
1-e_{01}=2 ; 2-e_{01}=5 ; 3-e_{01}=8 ; 4-e_{01}=12
$$
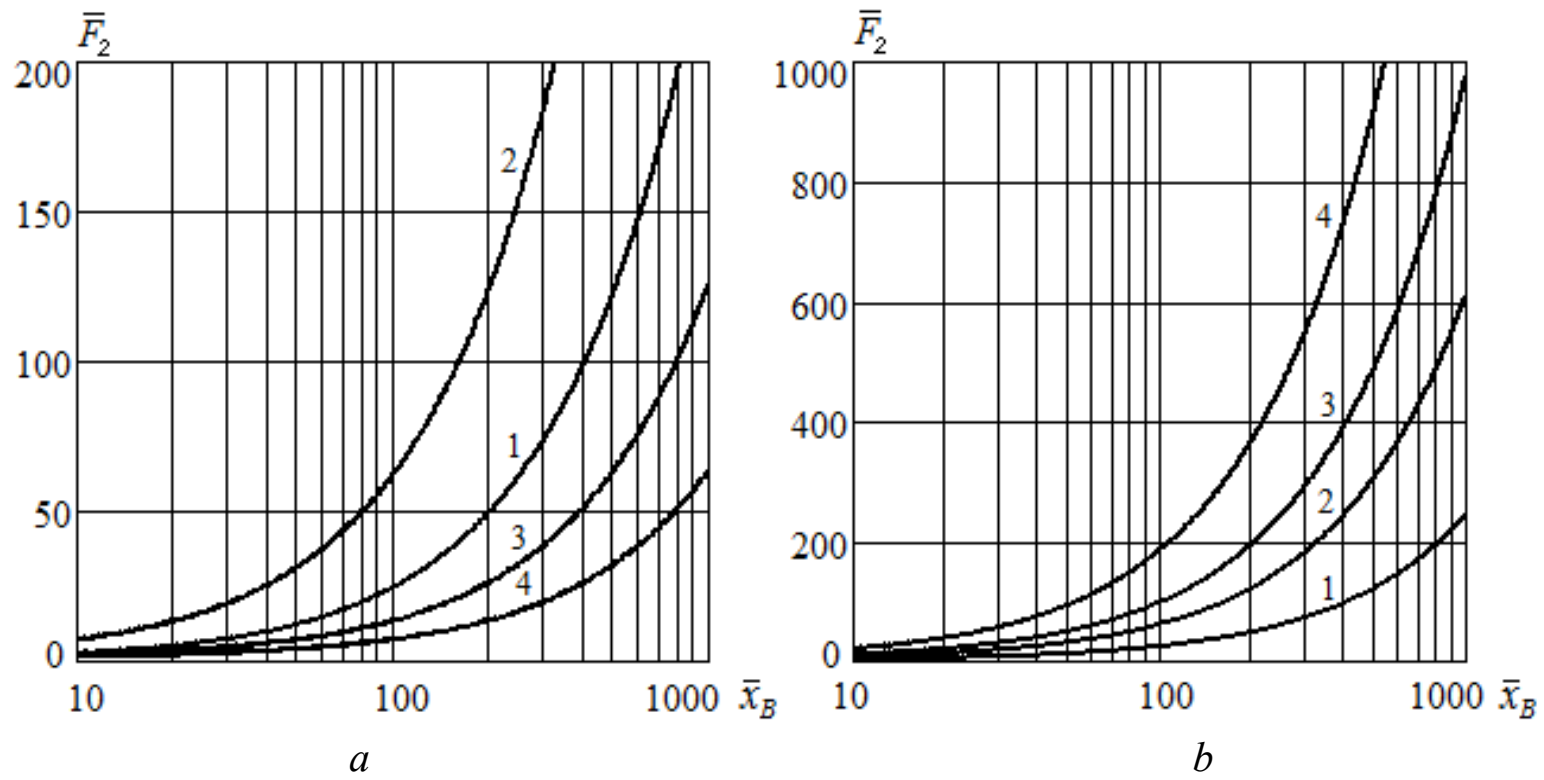

Figure 2. The dependence of the dimensionless friction force on the dimensionless length of the edge (scale $\bar{x}_{B}-$ logarithmic): 
$a$ - at various values of the dimensionless sliding speed $\left(e_{01}=5\right)$ :

$1-\bar{u}=0,01 ; 2-\bar{u}=1 ; 3-\bar{u}=5 ; 4-\bar{u}=10 ;$

$b-$ at different values of the measure of material elasticity $(\bar{u}=1)$ :

$$
1-e_{01}=2 ; 2-e_{01}=5 ; 3-e_{01}=8 ; 4-e_{01}=15
$$

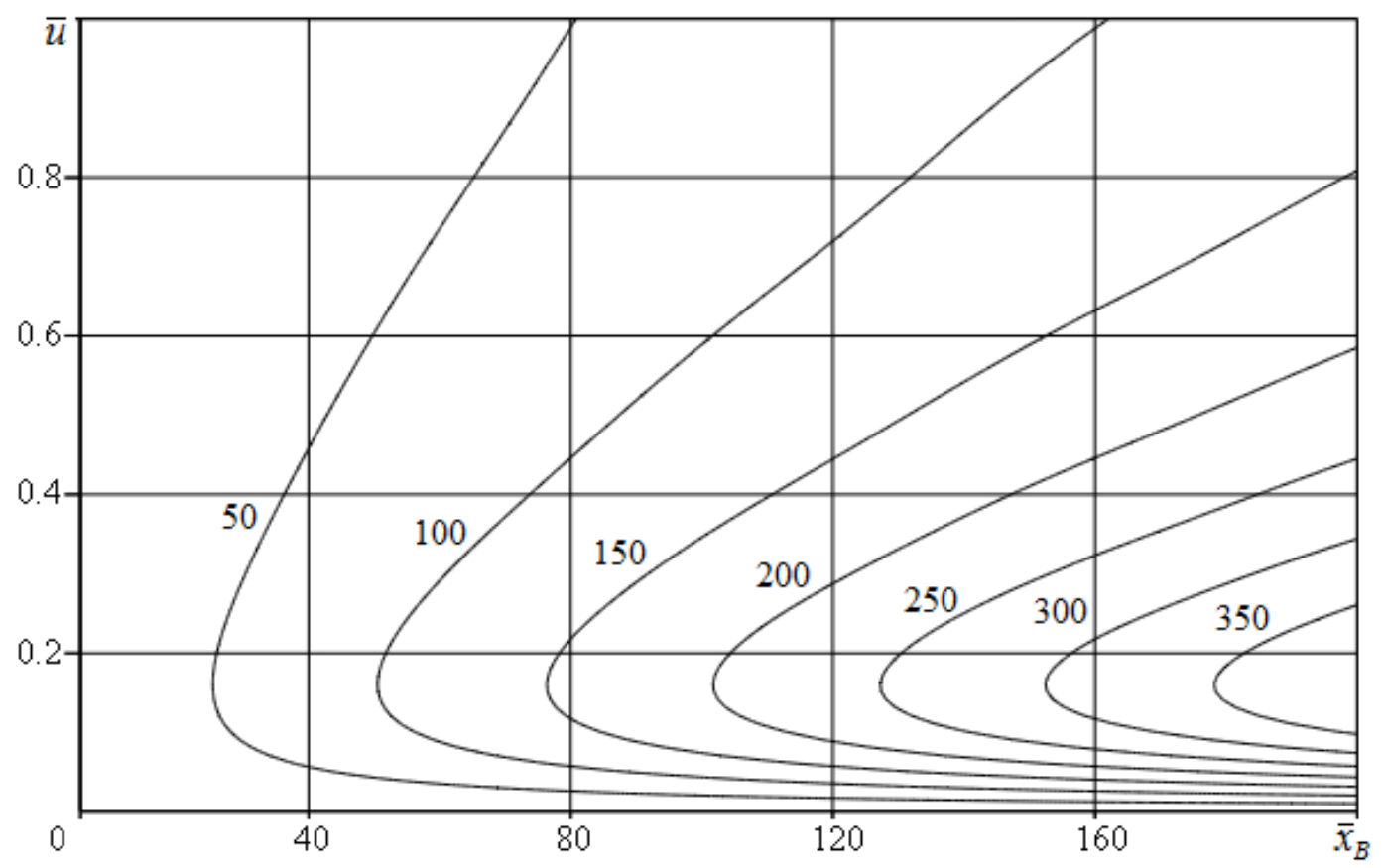

Figure 3. The dependence of the dimensionless friction force $\bar{F}_{2}$ on the dimensionless length of the edge and on the dimensionless sliding speed $\left(e_{01}=5\right)$

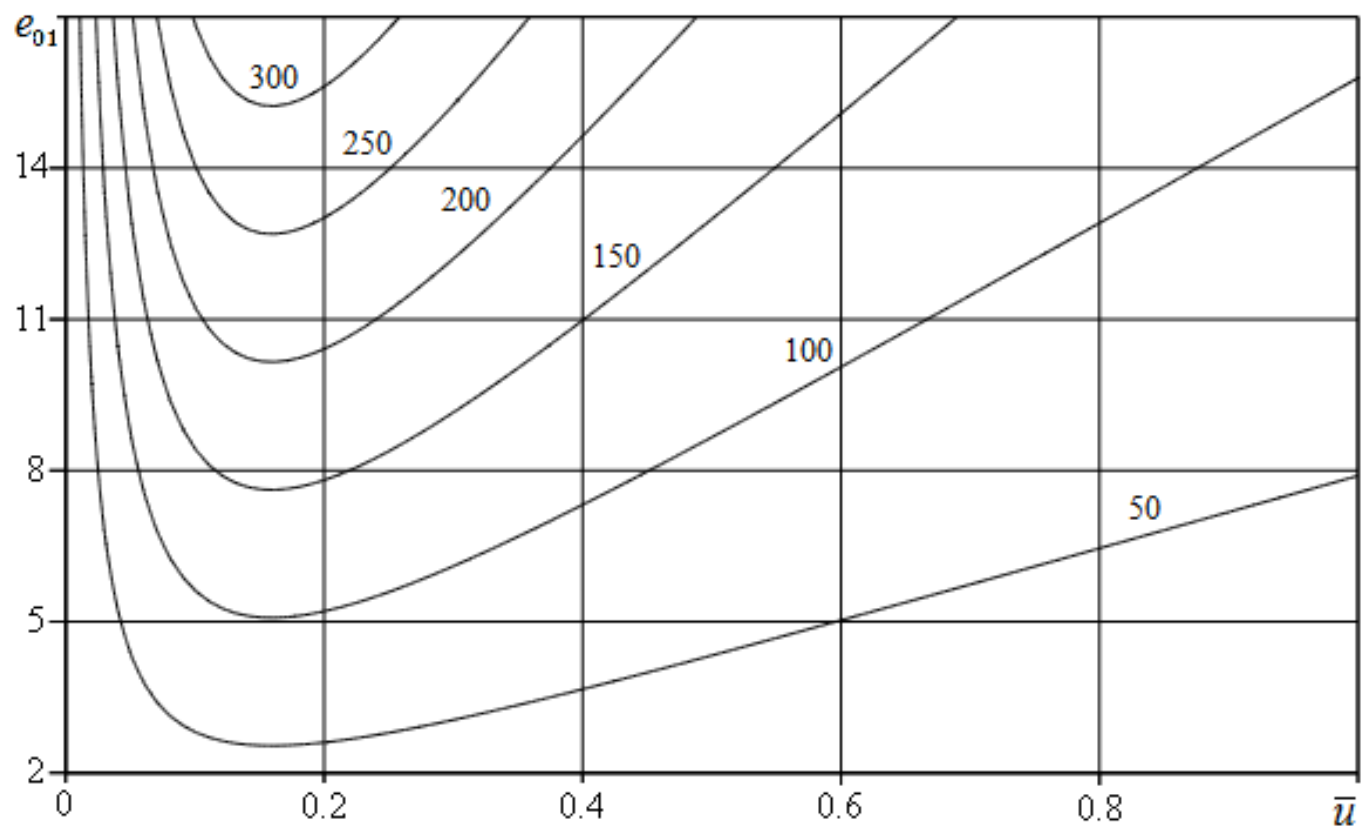

Figure 4. The dependence of the dimensionless friction force $\bar{F}_{2}$ on the dimensionless sliding speed and on the measure of material elasticity $\left(\bar{x}_{B}=50\right)$ 


\section{Conclusions}

The distribution of contact pressures and the deformation friction force of the knife substantially depend on the roughness shape of the rough surface of the edge, as well as on the sliding speed and the rheological properties of the food material. When a certain value of speed is reached, this force has a pronounced maximum, after which it nonlinearly decreases.

The dimensionless width of the contact area of the microprotrusions $x_{c}$ monotonously depends on the measure of the material elasticity and non-monotonously depends on the dimensionless sliding speed with a pronounced minimum.

The established dependences make it possible to scientifically manage the geometric shape of the unevenness of the technological roughness of a knife in order to minimize the deformation friction force when cutting food materials.

\section{References}

Ageev, O.V., Naumov, V.A., Fatykhov, Yu.A., Samoilova, N.V. (2018) Correspondence of rheological models to the structuralmechanical properties of fish.

Nauchnyy zhurnal Sankt-Peterburgskogo nacional'nogo issledovatel'skogo universiteta informacionnyh tehnologiy, mehaniki $i$ optiki. Processy $i$ apparaty pishhevyh proizvodstv, 2(36), 34-43.

Belaasilia, Y., Braikat, B., Jamal, M. (2017). High order mesh-free method for frictional contact. Engineering Analysis with Boundary Elements, 82, 68-78.

Belaasilia, Y., Timesli, A., Braikat, B., Jamal, M. (2018). A numerical mesh-free model for elasto-plastic contact problems. Engineering Analysis with Boundary Elements, 94, 103112.

Deibel, K-R., Raemy, C., Wegener, K. (2014). Modeling slice-push cutting forces of a sheet stack based on fracture mechanics. Engineering Fracture Mechanics, 124-125,
234-247.

Grigoriev, A.Y. (2016). Physics and microgeometry of technical surfaces. Minsk, Belaruskaya navuka, 247 p.

Jadav, P.U., Amali, R., Adetoro, O.B. (2018). Analytical friction model for sliding bodies with coupled longitudinal and transverse vibration. Tribology International, 126, 240248.

Kieserling, K., Schalow, S., Drusch, S. (2018). Method Development and Validation of Tribological Measurements for Differentiation of Food in a Rheometer. Biotribology, 16, 25-34.

Schuldt, S., Schneider, Y., Rohm, H. (2018). High-speed cutting of foods: Cutting behavior and initial cutting forces. Journal of Food Engineering, 230, 55-62.

Spagnoli, A., Brighenti, R., Terzano, M., Artoni F. (2019) Cutting resistance of soft materials: Effects of blade inclination and friction. Theoretical and Applied Fracture Mechanics, 101, 200-206.

Spagnoli, A., Terzano, M., Brighenti, R., Artoni F., Ståhle P. (2018). The fracture mechanics in cutting: A comparative study on hard and soft polymeric materials. International Journal of Mechanical Sciences, 148, 554564.

Wang, P., Ni, H., Wang, R., Li, Zh., Wang, Y. (2016). Experimental investigation of the effect of in-plane vibrations on friction for different materials. Tribology International, 99, 237-247.

Winkeljann, B., Bussmann, A.B., Bauer, M.G., Lieleg, O. (2018). Oscillatory Tribology Performed With a Commercial Shear Rheometer. Biotribology, 14, 11-18.

Xiao-Ping Zhou, Liang Fu, Wang Ju, Berto F. (2019). An experimental study of the mechanical and fracturing behavior in PMMA specimen containing multiple 3D embedded flaws under uniaxial compression. Theoretical and Applied Fracture Mechanics, 101, 207-216. 